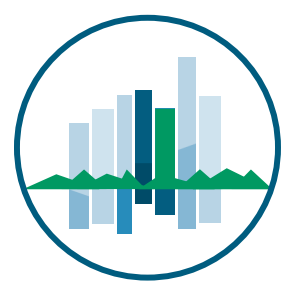

True Smart and Green City?

8th Conference of the

International Forum on Urbanism

Conference Proceedings Paper

\title{
Comparable Measures of Accessibility to Public Transport by the General Transit Feed Specification (GTFS)
}

\author{
Jinjoo Bok * and Youngsang Kwon \\ Department of Civil and Environmental Engineering, Seoul National University / 35-425, 1 Gwanak- \\ ro, Gwanak-gu, Seoul, South Korea \\ * Author to whom correspondence should be addressed; Tel.: +82-10-9092-2404; E-mail: \\ manjjew@gmail.com
}

\begin{abstract}
Public transport plays a critical role in the sustainability of urban settings. The mass mobility and quality of urban lives can be improved by establishing public transport networks that are accessible to pedestrians within a specific walking distance, which would also reduce monetary and environmental costs. Accessibility to public transport is the ease with which inhabitants can reach means of transportation such as buses, trams, metros, and trains. By measuring the degree of accessibility to public transport networks using a common data format, a comparative study can be conducted between competitive cities or metropolitan areas with different public transport systems. The General Transit Feed Specification (GTFS) by Google Developers allows this by offering a common format for public transportation stops, routes, trips, schedules, and associated geographic information in a series of text files. This paper suggests a method to measure the degree of accessibility to public transit in different urban areas using the GTFS, which is an open-source data set voluntarily produced and shared by the public transit agencies of many participating cities around the world. Functional Urban Area (FUA), which was identified by the OECD in 2013 for global comparative research, was applied as a spatial unit of analysis. Areas a maximum distance of approximately $330 \mathrm{~m}$ from each bus and tram node, and 660m from each metro and train node, were considered as Service Areas. These Service Areas were then classified into five levels according to operating frequency at each node to assess and compare the degrees of accessibility across FUAs. The analysis was carried out on the route networks of each urban area using ArcGIS 9.3. The results from eight FUAs show that Melbourne and Portland have higher degree of accessibility to public transport.
\end{abstract}


Keywords: Accessibility; Public Transport; Sustainability; GTFS

\section{Introduction}

Public transport has significant impacts on the economic performance and environmental quality of cities by facilitating mobility and enabling urban areas to function effectively. From the perspective of people's life in regions, access to works and services facilities is an important factor that shapes well-being, and public transport plays a crucial role to provide the access to those destinations.

Public transit can be conceived as one component making up the modal split such as walking, cycling, or vehicles (Matulin et al., 2009). Other than transport modes restricted for private use, such as the privately owned car or bicycle, public transport is distinguished by being open to a wider user group. As such it has the function of a public or common means enabling mobility. For the purpose of measuring accessibility, in this paper, public transport will be defined into two broader groups: rail-based (metro or train) and road-based (bus or tram).

The concept of accessibility has been understood and used in various ways (Gould, 1969 as cited in Makrí/Folkesson, 1999). This can already be noted by the terminological differences: terms as "access", "accessibility", "availability" or "proximity" are often used in exchangeable ways, referring to the same meaning (Murray et al., 1998; Bhat et al., 2005; Gomide et al., 2005). However, the term accessibility itself often takes quite different meanings because researchers and agencies adopt distinct perspectives, highlight different dimensions and pursue different goals and objectives in distinct contexts (Geurs/Wee, 2004; Handy/Niemeier, 1997). There are two broad sets of literatures in terms of accessibility in relation to public transportation:

- The first one, based on the classical "potential of opportunities" model (Hansen, 1959) considers transit accessibility merely as a means for reaching other urban services, such as health care, education or jobs. It is often referred to as network accessibility.

- The second and more recent one, on the other hand, defines transit accessibility as a crucial urban service to be reached. It is often referred to as local accessibility.

Notwithstanding the importance of network accessibility, this paper deals with local accessibility. Local accessibility could be framed by multiple dimensions including both quantitative and qualitative components. Given the data availability and the purpose of the research, international comparison, this paper focuses on the spatial and temporal components of accessibility of public transport. The spatial component of accessibility is expressed as the distance from a place of departure to a public transport. Physical distance to a transport facility, such as a bus stop or metro station is the most basic component of accessibility of public transport, as public transport means need to be physically reachable for urban residents. Yet, living physically close to public transport is not sufficient. In order to enable urban residents to pursue their daily travels, such as going to work, to school, to the doctor or shopping, it is crucial to ask when and how often public means of transport can be accessed. As for the distribution of transit modes, it has been common to compare the service volume during peak, with the average service volume per day (or as well as in other time units, such 
as per week or per month). The transport service offer also has been commonly indicated in terms frequency, i.e. how many times a certain transport mode can be accessed in a given timespan. It usually has been calculated as trips per hour, taking the average of scheduled headways, which can be obtained from timetable data of transport providers.

Monitoring and evaluating the performance of public transport requires indicators that effectively capture the relationship between land use and public transport in cities. Given the international urban context of growing metropolitan areas, more integrated way to measure and improve performance of public transport at the metropolitan level is required. In this regard, 1,179 Functional Urban Areas (FUAs) around the globe is eligible spatial units including urban cores and hinterlands according to the definition ${ }^{1}$ developed by OECD based on demands for the common spatial unit for comparative research on metropolitan areas (OECD, 2012).

This study aims to present the methodology for measuring accessibility to public transport in the internationally comparative way. In the context of current urban and regional policies, achieving sustainable urban form and improving regional well-being are key challenges, and public transport plays a vital role to respond to these issues. Measuring accessibility to public transport will clarify to what extent people have access to public means of transport for their daily life trips in metropolitan areas. It will also contribute to help policy makers to design policies to achieve sustainable urban form, and assess the policy repercussion.

\section{Results and Discussion}

Figure 2 and 3 provide geographic distribution of serviced areas according to the accessibility levels in the 8 metropolitan areas. Each map shows the functional metropolitan $\operatorname{area}^{2}$, both in terms of urban core (in white) and hinterland (grey). Both the size of the overall metropolitan area and population vary considerably across the FUAs (Figure 1). For the matter of frequency data availability, only serviced areas by each transit mode, not integrated accessibility levels, are expressed in cases of Toyama and Melbourne. The aggregated serviced areas differentiated according to accessibility levels from very high to no access (from dark to light shades displaying very high to low accessibility) are displayed. It shows that the highest levels of access concentrate around central nodes in the urban core areas, with lower levels radiating towards the margins of the urban core and spreading sparsely into the hinterlands. Despite considerable variation, a certain distribution pattern of larger population shares and higher levels in urban cores is observed in all assessed areas. In comparison to Asian and Australian metropolitan areas, north-American results illustrate more intensively concentrated catchment areas in a smaller portion of areas within urban cores, seemingly corresponding with the relatively extensive land use pattern and high dependency on automobiles.

\footnotetext{
1 The details of the definition of functional urban areas can be found here http://www.oecd.org/gov/regionalpolicy/Definition $\% 20$ of $\% 20$ Functional $\% 20$ Urban $\% 20$ Areas $\% 20 \% 28$ FUA $\% 29 \% 20$ for $\% 20$ the $\% 20$ OECD $\% 20 \mathrm{me}$ tropolitan\%20database.pdf

${ }^{2}$ Except for Great Melbourne, Australia, which is considered as a metropolitan area, however, not identified as FUA.
} 
Figure 1. Population in metropolitan areas, 2012.

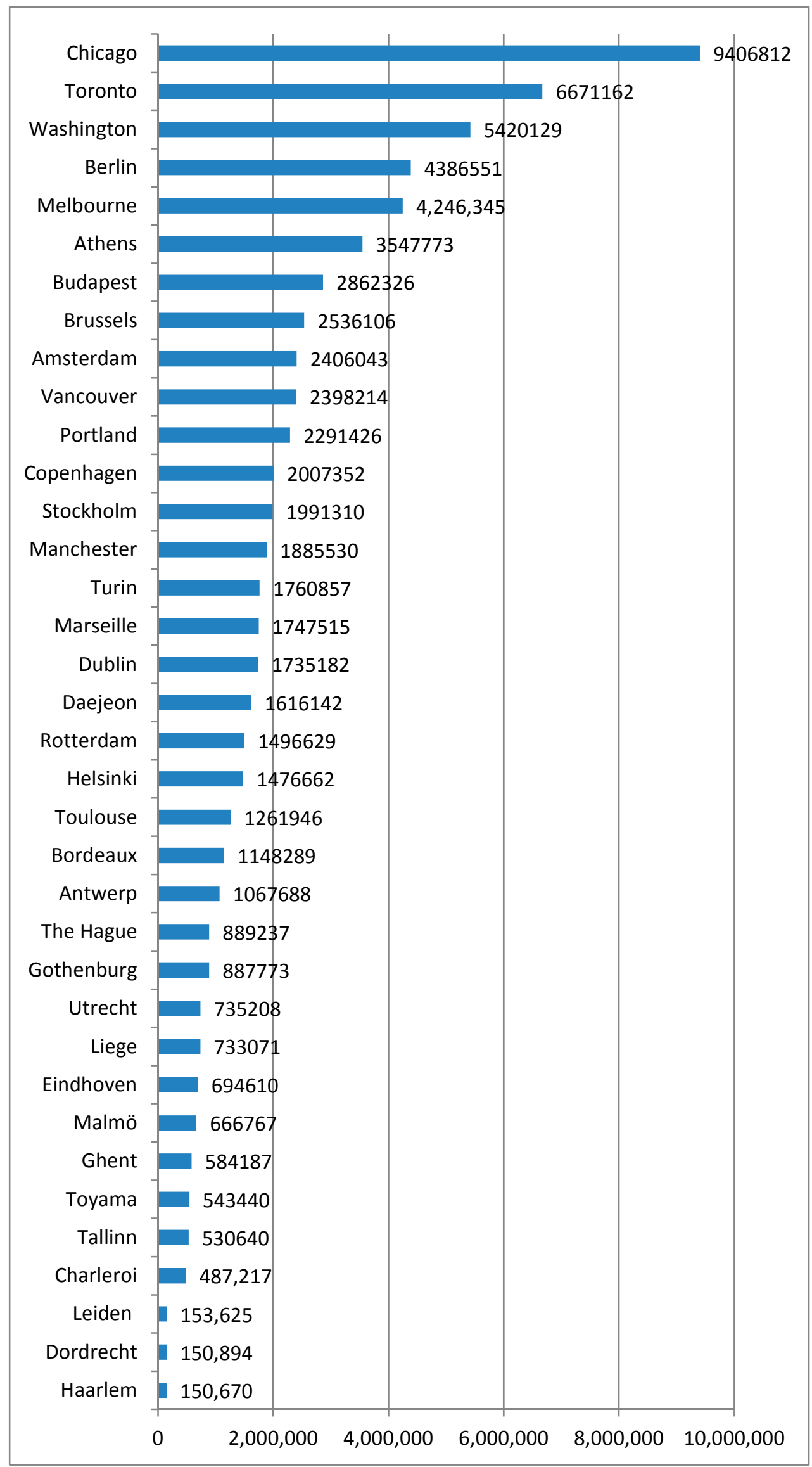

Source: OECD database and Eurostat. 
Figure 2. Serviced areas according to accessibility levels (Chicago, Portland, Washington, Toronto).
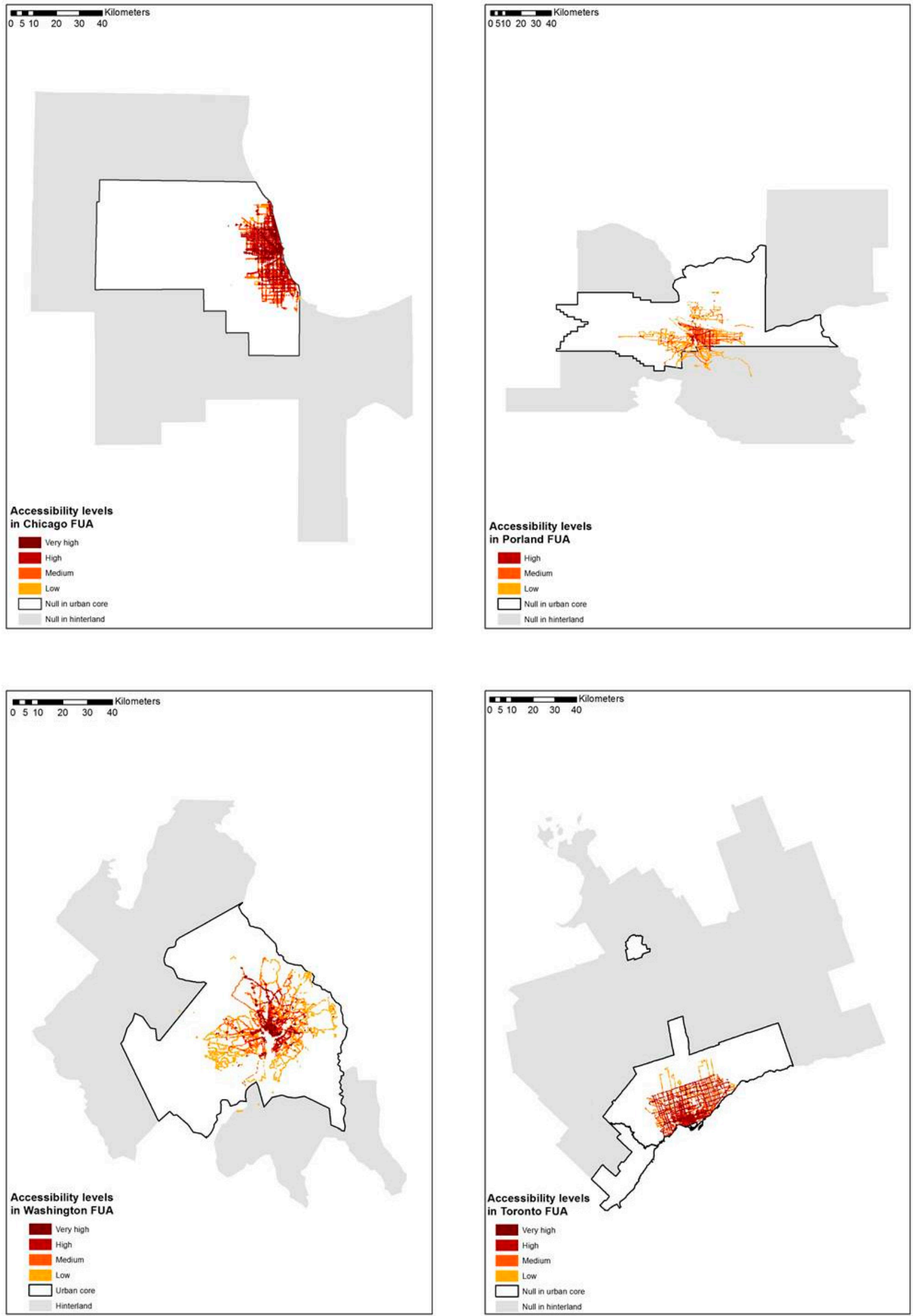
Figure 3. Serviced areas according to accessibility levels (Vancouver, Daejeon, Toyama, Melbourne)
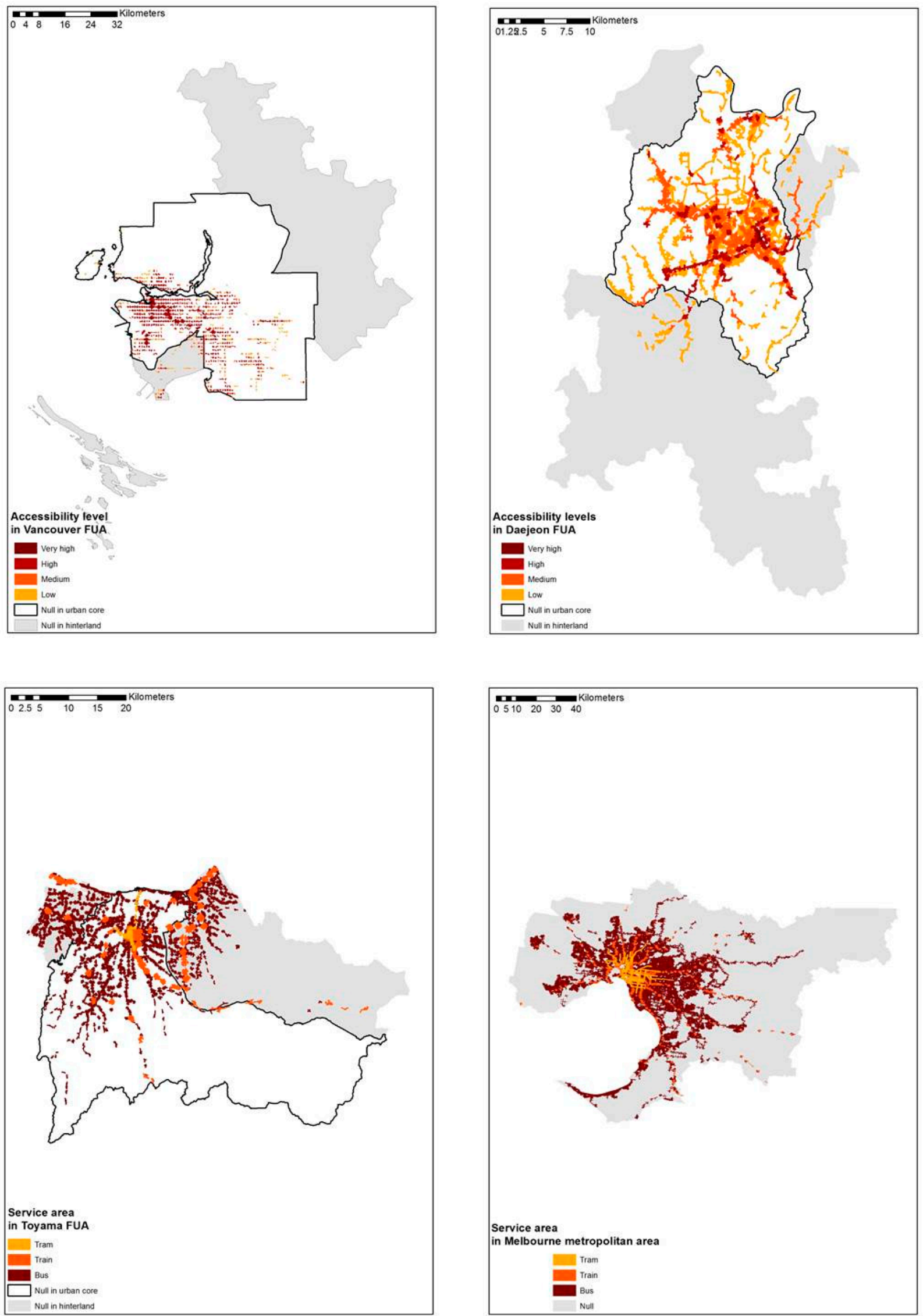
The share of population of serviced areas more clearly reveals the accessibility to public transport across the case regions, compared with another 23 European metropolitan cases. Figure 4 shows the share of population having access to public transit according to access levels for $6^{3}$ non-European cases and 23 European cases.

Figure 4. Share of population according to access levels.

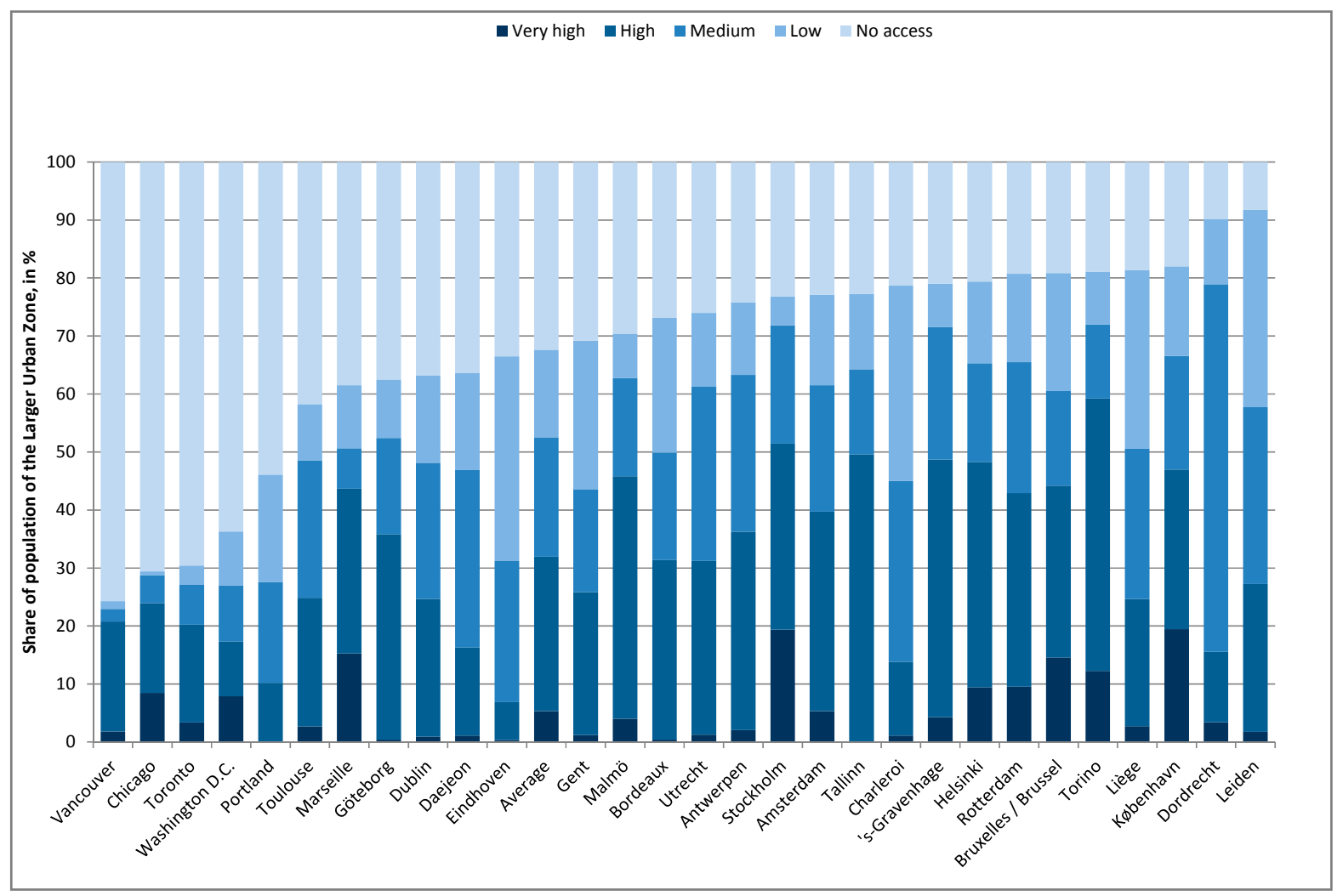

Source: modified based on EC calculations

\section{Experimental Section}

Based on conceptions and definitions above, measuring accessibility to public transport for metropolitan areas includes four steps: (1) Data collection; (2) identification of serviced areas; (3) classification of accessibility levels; and (4) calculation of population shares according to the levels. In cases of lacking data on frequency, only the share of population living in public transport serviced areas are calculated.

Step 1. Required datasets:

- Locations of public transport access points (stops and stations);

- The pedestrian street networks;

- The frequency of public transport (timetable data)

- The geographic distribution of population within a metropolitan area.

\footnotetext{
${ }^{3}$ Toyama and Melbourne are excluded for the lack of frequency data.
} 
Given the purpose of this study, currently available data source could be critical. As for North American and European areas, data on locations and frequency can be accessed and obtained via the GTFS project ${ }^{4}$ online in the common package including information on public transport stops, stations, routes, and timetables. On the other hand, as for Asian and Oceania areas, individual data should be collected through variable sources as the GTFS project has not covered those areas ${ }^{5}$. The geographic distribution of population within metropolitan areas is based on the population grid data per $1 \mathrm{~km}^{2}$ from the Landscan data (2009) for all regions.

\section{Step 2. Identifying serviced areas of public transport}

Serviced areas of public transport are created by combining data on locations of transit stops and the route networks through the network analysis tool of ArcGIS 9.3. Once overlaid, the served areas can be defined by calculating pedestrian walking time for pedestrians using the defined thresholds of 5 min walking time for accessing road-based transit (bus, tram), and 10min walking time for accessing rail-based transit (train, metro). As mentioned above, walking speed applied at this stage is $1.1 \mathrm{~m} / \mathrm{s}$ (almost $4 \mathrm{~km} / \mathrm{h}$ ) resulting into $330 \mathrm{~m}$ network distance to road-based transit stations, and $660 \mathrm{~m}$ network distance to rail-based transit stations.

\section{Step 3. Classification of accessibility of serviced areas}

The temporal component, frequency, is added for combined assessment after having defined serviced areas of public transport. As public transport modes are categorized into two wider modes, road-based and rail-based, there are two steps to finalize mode-integrated classification for each metropolitan area: (1) Classifying each serviced areas for each mode by frequency data; and (2) reclassification by aggregating mode-specific classes.

Frequency levels are determined by using the average amount of public transport services headways per hour. To avoid underestimating the effects of nearby stops on frequency, serviced areas within a perimeter of $50 \mathrm{~m}$ are combined and considered as a single area. The mode-specific frequency classes have been defined as follows and applied for both modes (Table 1).

Table 1. Classification of serviced areas by frequency.

\begin{tabular}{c|c}
\hline Class & Description \\
\hline High & $10<$ Average departure / hour (from 6 am to $8 \mathrm{pm}$ ) \\
\hline Medium & $4<$ Average departure / hour (from 6 am to $8 \mathrm{pm})=<10$ \\
\hline Low & $0<$ Average departure / hour (from 6 am to $8 \mathrm{pm})=<4$ \\
\hline Null & No operation \\
\hline
\end{tabular}

\footnotetext{
4 "The General Transit Feed Specification defines a common format for public transportation schedules and associated geographic information. GTFS "feeds" allow public transit agencies to publish their transit data and developers to write applications that consume that data in an interoperable way". https://developers.google.com/transit/gtfs/?hl=fr
}

\footnotetext{
${ }^{5}$ For this reason, any readily available data on frequency were not found for Toyama and Melbourne.
} 
Aggregated accessibility levels of public transport are divided into five classes (Table 2). While only the combination of high rail- and high road-based public transport results into very high level of accessibility, different combinations result into high, medium, low levels and null accessibility.

Table 2. Aggregated reclassification.

\begin{tabular}{l|l|l|l|l|l}
\hline \multicolumn{2}{c|}{} & \multicolumn{4}{l}{ Road-based mode (Bus and tram) } \\
\cline { 3 - 6 } \multicolumn{2}{c|}{ High } & Medium & Low & Null \\
\hline \multirow{3}{*}{$\begin{array}{l}\text { Rail-based } \\
\text { mode } \\
\begin{array}{l}\text { Train } \\
\text { metro) }\end{array}\end{array}$} & High & Very High & High & High & High \\
\cline { 2 - 6 } & Medium & High & Medium & Medium & Medium \\
\cline { 2 - 6 } & Low & High & Medium & Low & Low \\
\cline { 2 - 6 } & Null & High & Medium & Low & Null \\
\hline
\end{tabular}

Step 4. Calculating the share of population in transit access/service areas

By calculating the shares of population living in a catchment area of each accessibility level, comparison across metropolitan areas could be performed. As case areas, 8 FUAs are selected: Toyama (Japan); Daejeon (Korea); Melbourne (Australia); Chicago/Portland/Washington (U.S.); and Toronto/Vancouver (Canada). Then the results are compared with 23 European metropolitan areas in terms of accessibility of public transport ${ }^{6}$. In cases that frequency data are available, these shares are calculated according to the different service levels (ranging from very high to no service). For metropolitan areas not featuring the information, only the share of the population shares living in catchment areas are calculated.

\section{Conclusions}

Ensuring accessibility of public transport is an important task for sustainable urban development. This study focused on conceptualizing the integrated indicator and providing viable methodology and data sources for measuring and comparing accessibility of public transport for metropolitan areas. Whereas there are various ways in regards, this paper suggested applying spatial and temporal dimensions of accessibility based the common spatial unit of analysis for comparison of assessment results.

The analyzed results indicate how many people living in FUAs have relatively easy access to public transport, both in terms of physical access and the level of service frequency provided. Based on the output resulting from this methodology, the difference is striking between European and non-European cities, especially cities from North America. The general pattern of larger population shares being serviced in urban cores, as well as higher levels of service frequency within urban cores compared to the overall FUAs tends to confirm the intuition and knowledge of public transport development and land use patterns: the more densely inhabited urban areas tend to feature a better public transport than in hinterlands.

\footnotetext{
${ }^{6}$ European Commission, 2014
} 
Even though this study explored the challenges and opportunities of measuring accessibility of public transport in the internationally comparative way, there are still remaining limitations such as data coverage and the selection of sample cases. Although GTFS is even currently beneficial dataset voluntarily provided by local transport authorities and governments, it is still needed to be updated for better spatial coverage and accurate frequency.

\section{Acknowledgments}

This study was conducted as a Measuring Accessibility of Public Transport project in the OECD.

\section{References and Notes}

1. Bhat, C. R. et al. Metropolitan Area Transit Accessibility Analysis Tool; Centre for Transportation Research University of Texas: Texas, US, 2005.

2. Daniels, R.; C. Mulley. Explaining Walking Distance to Public Transport: the Dominance of Public Transport Supply. World Symposium on Transport and Land Use Research: Whistler Canada, 2011.

3. Geurs, K.T.; Wee, B. van. Accessibility Evaluation of Land-use and Transport Strategies: review and research directions. Journal of Transport Geography 2004, 12. 7-140.

4. Gomide, A. et al. Transport and Urban Poverty: A Synthetic Index of Adequate Service; The World Bank: Washington DC, US, 2005.

5. Handy, S. L.; D. A. Niemeier. Measuring Accessibility: an Exploration into Issues and Alternatives Environment and Planning 1997, 29(7), 1175-1194.

6. Hansen, W.G. How Accessibility Shapes Land Use. Journal of the American Institute of Planners $1959,25,73-76$.

7. Makrí, M.-C.; C. Folkesson. Accessibility Measures for Analyses of Land Use and Travelling with Geographical Information Systems; Department of Technology and Society. Lund Institute of Technology: Sweden: 1999, pp. 1-15.

8. Matulin, M., I. Bosnjak, L. Simunovic. Different Approaches to the Modal Split Calculation in Urban Areas; Faculty of Transport and Traffic Sciences: University of Zagreb, 2009.

9. Murray, A. T. et al. Public Transportation Access. Transport Research Part D: Transport and Environment 1998, 3(5), 319-328.

10. OECD. Redefining Urban: A New Way to Measure Metropolitan Areas; OECD Publishing: Paris, France, 2012.

(C) 2015 by the authors; licensee MDPI and IFoU, This article is an open access article distributed under the terms and conditions of the Creative Commons Attribution license. 